\title{
OBSERVATIONS PRÉLIMINAIRES SUR L'ATTAQUE DE QUELQUES ACIDES GRAS SUPÉRIEURS DE LA PART DE DIFFÉRENTES SOUCHES DE PÉNICILLIUM ROQUEFORTI
}

\author{
par \\ Pietro SALVADORI et Bruna Bianchi SALVADORI \\ CENTRO-SPERIMENTÁLE DEL LATTE - MILANO
}

\section{Introduction}

Ce travail se rattache à nos trois précédentes études expérimentales $[1,2,3]$ sur les caractéristiques protéolysantes de quelques souches de Penicillium roqueforti employées dans l'industrie laitière pour la fabrication de fromage Gorgonzola et persillés en général.

Cette fois-ci aussi nous avons pris en considération les trois souches fondamentales ayant des caractéristiques de protéolyse différentes :

Souche N : puissance protéolytique faible ;

Souche P : puissance protéolytique intermédiaire ;

Souche B : puissance protéolytique forte.

Ces souches utilisées dans la fabrication de fromages persillés confèrent au produit des caractéristiques de goût et commerciales différentes. Elles influent non seulement de façon différente sur le piquant du produit mais aussi sur l'intensité du persillage.

\section{But de la recherche}

Dans nos travaux précédents nous avions étudié le différent comportement des souches à l'égard de la protéolyse du lait et nous avions relevé des différences considérables tant du point de vue des produits de la protéolyse, que du différent comportement au, Warburg à l'égard de l'utilisation des divers acides aminés.

On sait bien que dans l'affinage des fromages se produisent des phénomènes de protéolyse (particulièrement violents dans le Gorgonzola), auxquels s'en ajoutent d'autres d'origine lipolytique qui, comme nous savons, donnent lieu à la formation d'acides gras libres et de cétones.

Considérant la protéolyse comme la seule caractéristique distinctive entre les souches que nous avons étudiées, nous avons voulu 


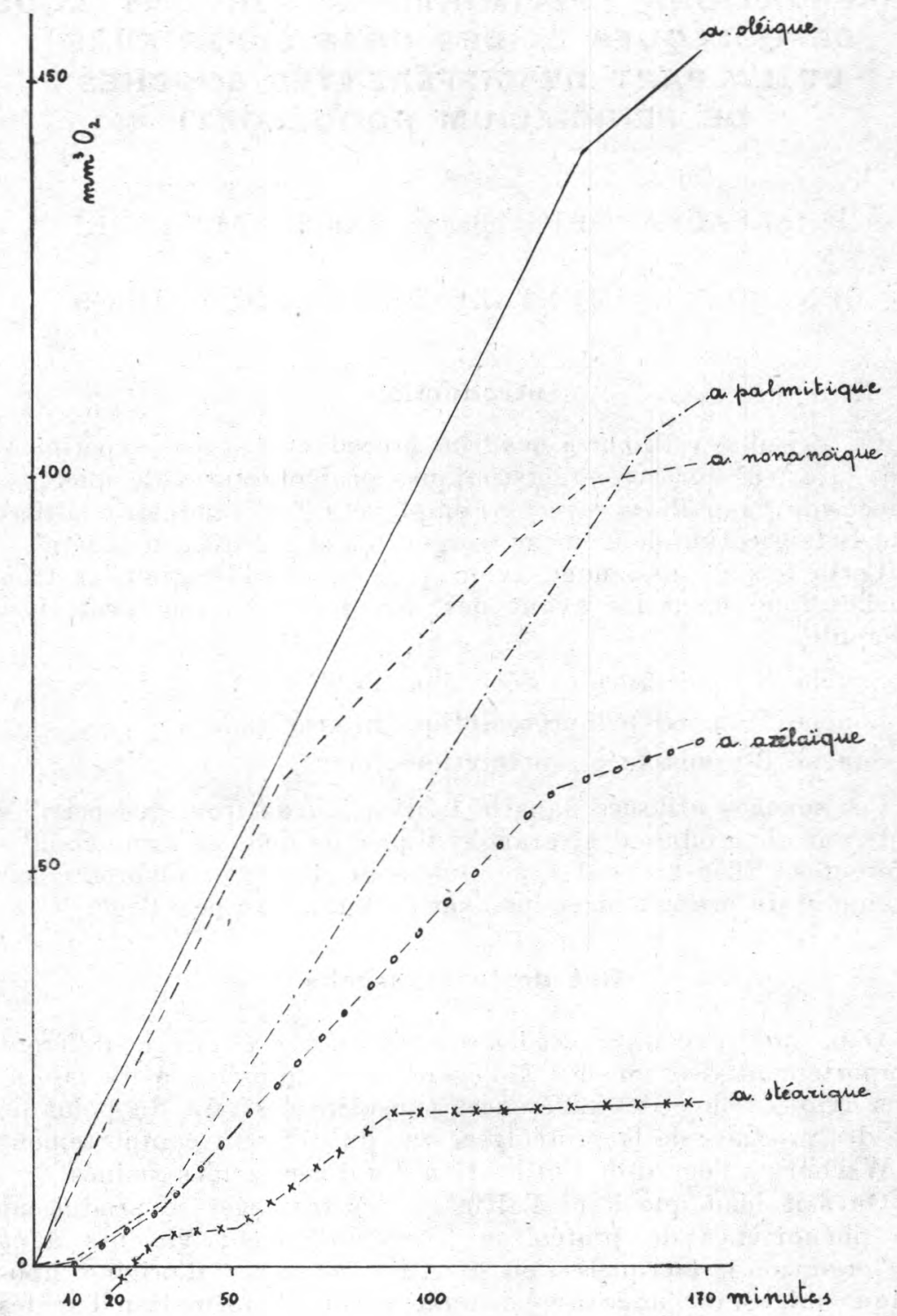

Fig. 1. - Consommation d' $\mathrm{O}_{2}$ du mycélium de la souche $\mathrm{N}$ en présence de 3 micro-moles des acides gras. 


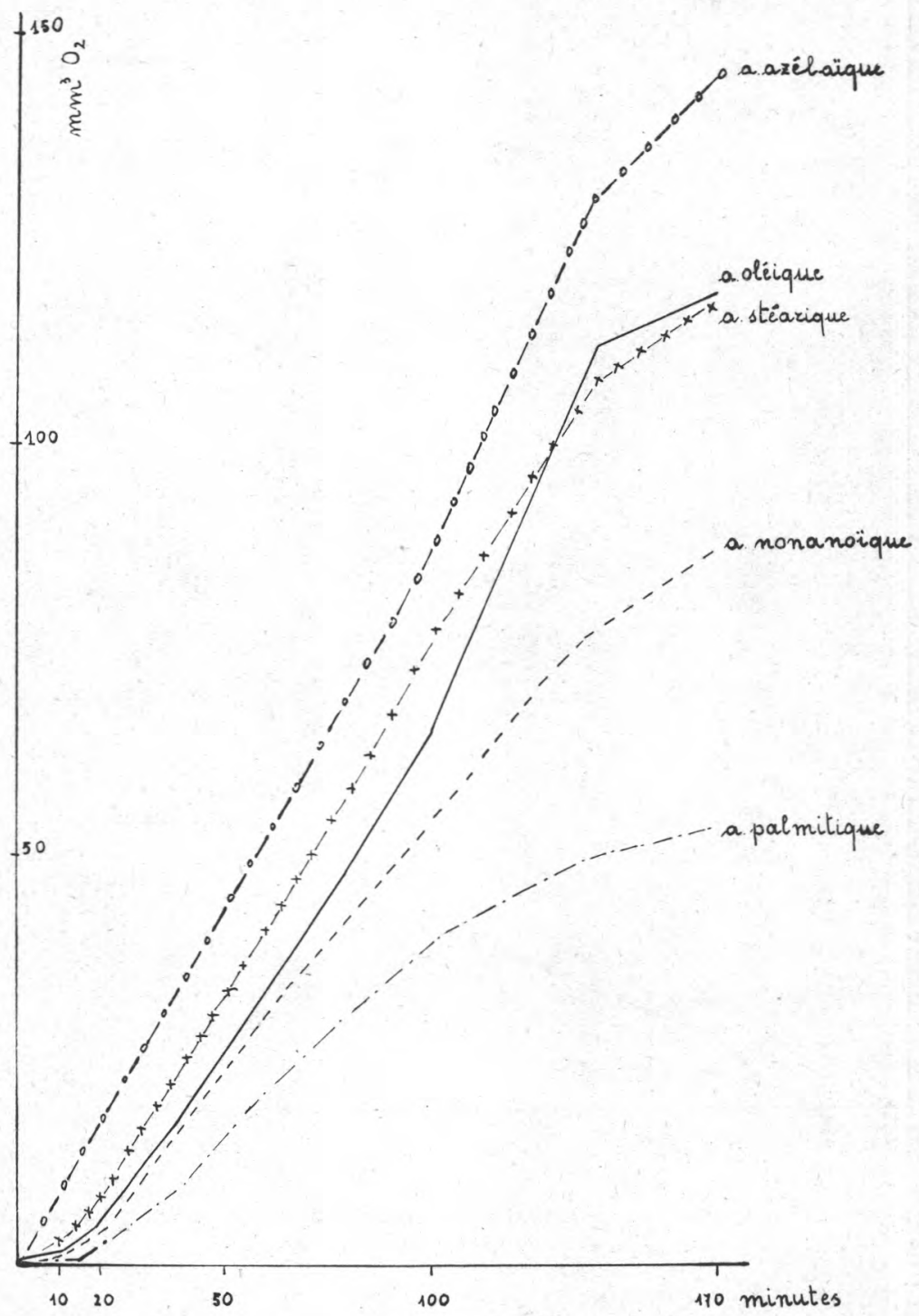

Fig. 2. - Consommation d' $\mathrm{O}_{2}$ du mycélium de la souche $\mathrm{P}$ en présence de 3 micro-moles des acides gras. 


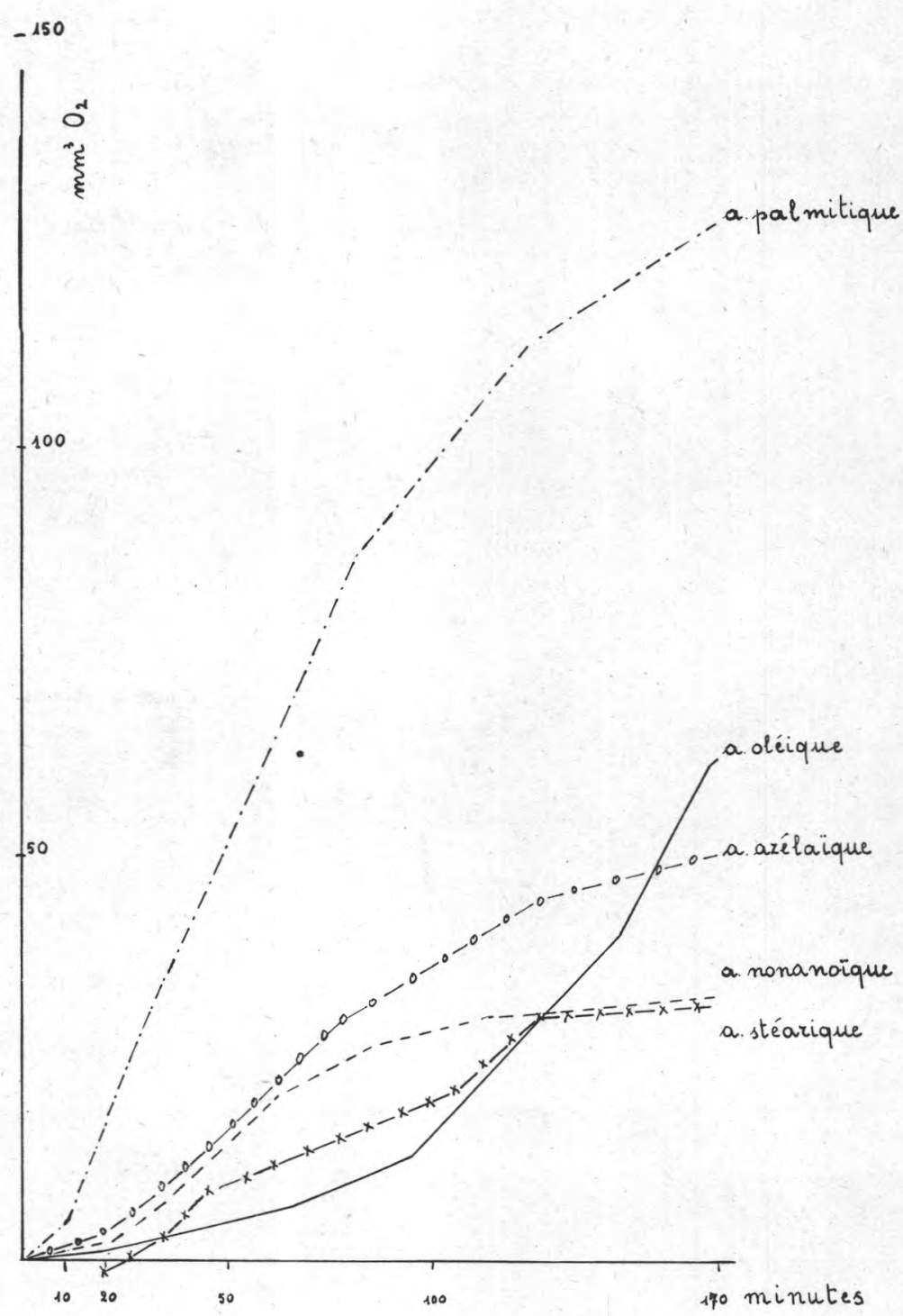

Fig. 3. - Consommation d' $\mathrm{O}_{2}$ du mycélium de la souche $\mathrm{B}$ en présence de 3 micro-moles des acides gras.

établir si ces mêmes souches se comportaient différemment aussi en attaquant quelques acides gras supérieurs, stéarique, oléique, palmitique, présents dans le gras du lait. 
Notre but est de démontrer si à une puissance protéolytique différente des moisissures correspond une égale puissance lipolytique [4].

Notre travail n'entend pas rechercher les produits d'origine enzymatique, mais seulement en montrer les effets, c'est-à-dire voir s'il existe effectivement une capacité intrinsèque d'attaquer les acides gras supérieurs énoncés précédemment. On sait que l'acide nonanoïque et l'acide azélaïque tirent leur origine de l'acide oléique, par oxydation. Dans notre étude nous avons voulu observer si les trois souches de Penicillium à l'examen avaient la capacité d'attaquer l'acide oléique et ses produits d'oxydation. Par conséquent nous avons effectué des essais au Warburg en mettant à l'épreuve nos trois souches avec de l'acide oléique, nonanoïque et azélaïque. En outre on a jugé opportun d'étudier sous ce point de vue aussi l'acide palmitique qui, comme on sait, dérive de l'acide oléique quand celui-ci se trouve en milieu alcalin. Enfin on a conduit les mêmes examens sur l'acide stéarique.

\section{Méthodes}

Le mycélium employé pour les expériences au Warburg était obtenu en maintenant en agitation des cultures de Penicillium en bouillon à la température de $25^{\circ} \mathrm{C}$ pendant 60 heures. Le mycélium recueilli sur Büchner était lavé de façon répétée avec un tampon phosphatique à $p H \quad 7,3$; ensuite, après avoir été séché, il était resuspendu en tampon phosphatique à raison de 2 p. 100. La suspension ainsi préparée était homogénéisée avec un appareil Ultraturrax (24000 tours/minute) pendant 10 secondes.

Dans chaque fiole du Warburg était introduite une quantité de suspension mycéliaire telle à avoir une teneur en azote totale de $0,30-0,40 \mathrm{mg}$.

Les acides gras étaient introduits, dans le diverticule approprié de la fiole, sous forme de sel sodique à raison de trois micro-moles par fiole. Afin d'éviter des concentrations trop faibles ou toxiques, on a procédé à un essai préliminaire pour établir la concentration idéale qui s'est avérée être trois miero-moles par fiole.

Les déterminations au Warburg ont été exécutées à la température de $25^{\circ} \mathrm{C}$.

Pour les méthodologies au Warburg nous renvoyons aux textes spécialisés [5].

Les valeurs relatives à la respiration endogène ont été soustraites des valeurs représentées dans les graphiques (fig. 1, 2 et 3).

\section{Résultats}

D'après la figure 1 nous pouvons remarquer que la-souche $\mathrm{N}$ attaque immédiatement l'acide oléique et l'acide nonanoïque, toutefois la consommation de $\mathrm{O}_{2}$ est plus prolongée dans le temps 
dans le cas de l'acide oléique comme le montrent les chutes des deux courbes. L'acide palmitique et l'acide azélaïque montrent une période considérable de latence après laquelle l'acide palmitique est oxydé plus que l'acide azélaïque.

Pour l'acide stéarique la consommation de $\mathrm{O}_{2}$ est de peu plus élevée par rapport à celle de la respiration endogène ; même au début, elle est inférieure.

Dans la figure 2 nous notons que la souche $\mathrm{P}$ utilise immédiatement l'acide azélaïque; l'acide oléique et l'acide stéarique sont attaqués après une période de latence de $15 \mathrm{mn}$, l'acide nonanoïque après une période de latence est oxydé dans une mesure inférieure aux deux derniers acides. Pour l'acide palmitique la période de latence est beaucoup plus prolongée.

Comme le montre la figure 3 , on a par la souche B une forte utilisation de l'acide palmitique; l'acide azélaĭque et nonanoïque sont faiblement utilisés après une période de latence de $40 \mathrm{mn}$; concernant l'acide oléique la période de latence atteint $100 \mathrm{mn}$; pour l'acide stéarique nous avons une oxydation négative pendant les 25 premières $\mathrm{mn}$, suivie d'une faible oxydation positive de peu supérieure à l'endogène.

\section{Conclusions}

Avant tout nous notons que dans l'oxydation des acides gras de la part des trois souches de Penicillium, on a des différences considérables.

Dans la souche $\mathrm{N}$ nous avons noté l'attaque de l'acide oléique d'où nous pouvons déduire la formation d'acide nonanoïque et azélaïque: D'autre part l'attaque immédiate de l'acide nonanoïque peut faire supposer une utilisation successive de cet acide tandis que l'acide azélaĩque, qui est faiblement utilisé, serait accumulé. Le long stade de latence à l'égard de l'acide palmitique et l'attaque négative de l'acide stéarique, qui à eux seuls forment 40 p. 100 des acides gras présents dans le lait, nous montrent qu'à la faible puissance protéolytique de la souche $\mathrm{N}$ répond une puissance lipolytique également faible.

Nous rencontrons la différence entre les souches $\mathrm{N}$ et $\mathrm{P}$ dans l'attaque de la part de la souche $\mathbf{P}$ de l'acide azélaïque qui par contre n'est pas utilisé par la souche $\mathrm{N}$. Au contraire, toujours par la souche $\mathbf{P}$, nous avons une faible utilisation de l'acide nonanoïque après une phase d'adaptation plutôt longue. On suppose done que la lipolyse de la part de la souche $P$, après l'attaque de l'acide oléique, suit la voie acide oléique acide azélaïque, tandis que la lipolyse de la souche $\mathrm{N}$ suivrait la voie acide oléique acide nonanoïque. Nous remarquons en outre une plus grande utilisation de la part de la souche $\mathrm{P}$ de l'acide stéarique par rapport à la souche $\mathrm{N}$.

La souche B diffère des deux autres souches par l'utilisation immédiate de l'acide palmitique qui, comme nous le savons, se 
trouve en plus grand pourcentage dans le gras du lait. Ceci justifie une plus grande puissance protéolytique qui s'accompagne d'une plus grande puissance lipolytique.

Nous avons l'intention de toute façon, vu ces résultats, de rechercher les produits dérivant de l'attaque des acides gras étudiés de la part des souches $\mathrm{N}, \mathrm{P}$ et $\mathrm{B}$.

\section{Résumé}

Les auteurs ont essayé d'établir si des souches de Penicillium ayant des caractéristiques de protéolyse différentes possédaient aussi des capacités lipolytiques différentes. On a effectué des essais au Warburg en mettant à l'épreuve les souches de Penicillium à l'examen avec les acides gras suivants : palmitique, oléique, stéarique, azélaïque et nonanoïque.

On a vu que les souches à l'examen utilisent de façon différente chaque acide gras d'où l'on peut déduire une différence des caractéristiques lipolytiques.

\section{Summary}

An attempt was made to find out whether Penicillium strains with different proteolysing potentials would exhibit different lipolysing properties, too.

Warburg tests were performed in which the Penicillium strains were tried on Palmitic, Oleic, Stearic, Azelaic and Pelargonic acids, and it was noted that a different action was exerted by each strain on the individual fatty acids used for the experiments - which appears to indicate differences in their lipolytic properties.

\section{RÉFÉRENCES}

[1] Salvadori (P.), Bianchi (B.) et Cavalli (V.). XVIe Congrès international laitier, vol. B p. 455-463, 1962.

[2] Salvadori (P.), Bianchi (B.) et Cavalli (V.). Il latte, I, p. 18-22, 1962.

[3] Salvadori (P.), Bianchi (B.) et Cavalli (V.). Le Lait, tome Xliv, p. $129-138,1964$.

[4[ Niki (T.), Yoshioнa (Y.) et Aнгко (K.). XVIIe Congrès international de laiterie, vol. D p. 531-537, 1966.

[5] Umbreit, Burris et Stauffer, Manometric Techniques, 1959. Burgess. 